\title{
Transtendon rotator-cuff repair of partial- thickness articular surface tears can lead to medial rotator-cuff failure
}

This article was published in the following Dove Press journal:

Open Access Journal of Sports Medicine

25 June 2014

Number of times this article has been viewed

\author{
Tom CWoods ${ }^{4}$ \\ Michael J Carroll' \\ Atiba A Nelson ${ }^{2}$ \\ Kristie D More ${ }^{2}$ \\ Randa Berdusco' \\ Stephen Sohmer ${ }^{3}$ \\ Richard S Boorman ${ }^{1,2}$ \\ Ian KY Lo ${ }^{1,2}$ \\ 'Department of Surgery, ${ }^{2}$ Sport \\ Medicine Centre, University of \\ Calgary, Calgary, AB, Canada; \\ ${ }^{3}$ Department of Orthopaedics, \\ University of British Columbia, \\ Vancouver, ${ }^{4}$ St Joseph's Hospital, \\ Comox, BC, Canada
}

Purpose: The purpose of this study was to evaluate clinical and anatomic outcomes of patients following transtendon rotator-cuff repair of partial articular supraspinatus tendon avulsion (PASTA) lesions.

Patients and methods: Patients in the senior author's practice who had isolated PASTA lesions treated by transtendon rotator-cuff repair were included $(n=8)$ and retrospectively reviewed. All patients were evaluated preoperatively and at a mean of 21.2 months ( \pm 9.7 months) postoperatively using standardized clinical evaluation (physical exam, American Shoulder and Elbow Surgeons, and Simple Shoulder Test). All patients underwent postoperative imaging with a magnetic resonance imaging arthrogram.

Results: There was a significant improvement in American Shoulder and Elbow Surgeons (42.7 \pm 17.5 to $86.9 \pm 25.2)$ and Simple Shoulder Test (4.6 \pm 3.2 to $10.1 \pm 3.8)$ scores from pre- to postoperative, respectively. Postoperative imaging demonstrated full-thickness medial cuff tearing in seven patients, and one patient with a persistent partial articular surface defect.

Conclusion: Transtendon repair of PASTA lesions may lead to improvements in clinical outcome. However, postoperative imaging demonstrated a high incidence of full-thickness rotator-cuff defects following repair.

Keywords: rotator cuff, PASTA lesion, transtendon repair

\section{Introduction}

Partial-thickness tears of the rotator cuff are common pathologic lesions, with prevalence increasing with age. ${ }^{1,2}$ Partial-thickness rotator-cuff tears have been classified according to location (ie, bursal surface, articular surface, or intratendinous), and further subclassified based on their depth. ${ }^{3}$ The acronym PASTA (partial articular supraspinatus tendon avulsion) lesion was introduced by Snyder, ${ }^{3}$ and despite improvements in diagnosis (by magnetic resonance imaging [MRI] or arthroscopy), treatment remains controversial. ${ }^{4}$

In patients with PASTA lesions involving $>50 \%$ of the tendon thickness or with $>6 \mathrm{~mm}$ of footprint exposed, surgical repair of the tendon is generally recommended over simple debridement of the lesion. ${ }^{5-8}$ Options for surgical repair include completion of the tear followed by standard rotator-cuff repair or via transtendon rotator-cuff repair. ${ }^{9-15}$ Transtendon rotator-cuff repair maintains the intact lateral portion of the tendon while repairing the medial aspect of the tendon back to the footprint. Theoretical benefits of a transtendon repair include anatomic restoration of the footprint and maintenance of the normal intact lateral cuff, which may improve biological or biomechanical characteristics and enhance healing. Recent studies have
Correspondence: lan KY Lo

Department of Surgery, University of Calgary, 3280 Hospital Drive Northwest, Calgary, AB T2N 4Z6, Canada

Tel +l 4032202745

Fax +l 4032108197

Emailikylo@ucalgary.ca 
demonstrated that transtendon rotator-cuff repair can lead to improved clinical outcomes. ${ }^{11,14-19}$ However, the extent to which transtendon rotator-cuff repair restores normal footprint anatomy is unclear.

The purpose of this study was to evaluate the clinical outcome and anatomic features of a small consecutive series of patients following transtendon rotator-cuff repair of PASTA lesions. We hypothesized that patients would show improvement in clinical outcomes and demonstrate restoration of footprint anatomy postoperatively.

\section{Patients and methods \\ Patients}

The study was designed as a retrospective consecutive case series. The University of Calgary Conjoint Health Research Ethics Board approved this research. Fourteen patients, over a 3-year period, were identified as having isolated PASTA lesions treated by transtendon rotator-cuff repair. All patients consented to participate in the study. Any patients requiring a significant concomitant procedure (eg, bicep tenodesis, SLAP [superior labral tear from anterior to posterior] repair, acromioplasty) were excluded from this study. Patients failing standard nonoperative treatment (eg, physical therapy, injections, home exercise program) and willing to participate in follow-up were included. Eight patients had complete clinical and radiographic follow-up, and were included in the final analysis. Four patients had incomplete clinical evaluation, and two refused a postoperative magnetic resonance imaging (MRI) arthrogram (MRA) and were not included in the current study. There was one female and seven male patients with a mean age of 52.2 \pm 8.7 years, with one nondominant and seven dominant extremities. The mean duration of symptoms was $11.0 \pm 5.8$ months. Six patients described a traumatic onset of symptoms, while two patients had an insidious onset of shoulder pain.

All patients underwent pre- and postoperative standardized clinical examination (American Shoulder and Elbow Surgeons [ASES] shoulder index score ${ }^{20}$ [score range is 10-100] and Simple Shoulder Test $[\mathrm{SST}]^{21}$ [score range is 0-12]). A simple questionnaire was administered postoperatively to determine the rate of patient satisfaction with the procedure, based on a 10-point ordinal scale. In addition, the patients were asked whether they would choose to have surgery again, based on a yes/no response. Patient demographic information and results are presented in Table 1.

\section{Surgical technique}

The transtendon-repair technique has previously been reported. ${ }^{14}$ In brief, arthroscopic assessment was used to
Table I Baseline characteristics of all patients $(n=8)$

\begin{tabular}{lll}
\hline & Mean & \pm \\
\hline Age (years) & 52.2 & 9.3 \\
Size of tear (mm) & 15.9 & 7.62 \\
Duration of symptoms (months) & 11.0 & 5.8 \\
Preoperative ASES score & 42.7 & 17.5 \\
Postoperative ASES score & 86.9 & 25.2 \\
Preoperative SST score & 4.6 & 3.2 \\
Postoperative SST score & 10.1 & 3.8 \\
Satisfaction score & 8.9 & $\mathrm{NA}$ \\
& Count & $\%$ \\
\hline Sex - male & 7 & 0.87 \\
Sex - female & $\mathrm{I}$ & 0.13 \\
Onset - traumatic & 6 & 0.75 \\
Onset - insidious & 2 & 0.25 \\
Dominant side - involved & 7 & 0.87 \\
Dominant side - not involved & $\mathrm{I}$ & 0.13 \\
\hline
\end{tabular}

Abbreviations: ASES, American Shoulder and Elbow Surgeons; SST, Simple Shoulder Test.

confirm an articular surface partial-thickness tear. The rotator cuff was debrided to viable healthy tissue, and the bone bed was debrided to a bleeding bone surface to enhance healing. The arthroscope was then inserted into the subacromial space, where a bursectomy was performed. A formal bony acromioplasty was not performed in these patients with articular surface rotator-cuff tears, which is the senior author's preference. The bursal surface of the rotator cuff was carefully assessed to rule out significant bursal surface tearing or a complete rotator-cuff tear. By visual estimation, if approximately 50\% or more of the tendon remained attached to the footprint of the rotator cuff, a transtendon repair was undertaken. The mean percentage of footprint involved was $62.1 \% \pm 18.1 \%$. Patients with less than $50 \%$ of tendon thickness involved were treated by debridement, and were not included in the current study. ${ }^{6,8}$ The arthroscope was reintroduced into the glenohumeral joint, and an 18-gauge needle was used as a guide for anchor trajectory, ensuring that the anchor would be placed at a deadman's angle of $45^{\circ}$ or less. Anchors $(5.5 \mathrm{~mm}$ Bio-Corkscrew $^{\circledR}$; Arthrex, Inc., Naples, FL, USA) were then placed percutaneously through a small stab incision lateral to the acromion, paralleling the 18-gauge needle, and inserted into the humeral head along the medial aspect of the footprint. If the tear involved greater than $1.5 \mathrm{~cm}$ in the anteroposterior dimension, a second anchor was inserted in the posterior medial aspect of the exposed footprint. Absorbable monofilament suture (PDS ${ }^{\circledR}$; Ethicon, Somerville, NJ, USA) was shuttled through the tendon using a spinal needle to form a horizontal mattress pattern. Sutures were then tied in the subacromial space using a Sixth Finger (Arthrex) compressing the tendon against the medial aspect of the footprint. 
Patients underwent a standardized rehabilitation program involving 4-6 weeks of sling immobilization, with early active hand, wrist, and elbow motion. Passive externalrotation was allowed immediately and forward elevation was initiated 3-4 weeks post-operatively. At 6 weeks, progressive range of motion was allowed with isotonic strengthening at 12 weeks. Strengthening exercises were instituted at approximately 10-12 weeks. By 6-12 months, unrestricted activities were permitted.

\section{Clinical and radiographic evaluation}

All patients underwent a standardized clinical examination, including ASES and SST scores by an independent evaluator blinded to the results of the follow-up imaging study. At final follow-up, all eight patients underwent a postoperative MRA. MRA is not routinely performed at our institution for clinical follow-up, but was included in this retrospective study to evaluate the anatomic outcome of transtendon repairs of partial articular surface rotator-cuff tears.

Prior to MRI, injection of contrast material was performed under fluoroscopic imaging via an anterior approach. The intra-articular position of the 20-gauge needle was confirmed, and $10-15 \mathrm{~mL}$ of gadopentetate dimeglumine (Magnevist ${ }^{\mathbb{R}}$; Bayer Leverkusen, Germany) in a 1:200 solution with normal saline was injected.

MRI (1.5 T MAGNETOM ${ }^{\circledR}$ Avanto; Siemens, Munich, Germany) was immediately performed after injection of contrast material. Patients were imaged with the arm in neutral position. The following sequences were routinely obtained: T1-weighted coronal fat suppression (FS), coronal T2 FS, axial T1 FS, axial T2, sagittal T1 FS, sagittal T2.

All MRA images were performed and read by a dedicated musculoskeletal radiologist. The radiologist was blinded to the patient's specific operative procedure and clinical outcome.

\section{Statistics}

Paired two-tailed Student's $t$-tests were used to compare preoperative and postoperative outcome scores. All statistical analyses were performed using SPSS (IBM, Armonk, NY, USA).

\section{Results Clinical outcome}

At a mean follow-up of 21.2 months $( \pm 9.7$ months, range 12-36 months), seven of eight patients were satisfied with the procedure, and all eight would have the surgery again. There was a significant improvement in ASES scores from
$42.7 \pm 17.5$ preoperatively to $86.9 \pm 25.2$ postoperatively $(P=0.0002)$ and SST scores from $4.6 \pm 3.2$ preoperatively to $10.1 \pm 3.8$ postoperatively $(P=0.001)$. All patients had improvement in ASES and SST scores postoperatively, except one who was dissatisfied with the outcome of the procedure but would still undergo the surgery again. This patient was involved in an ongoing litigation claim. When asked to score their subjective satisfaction on a 10-point ordinal scale, scores averaged 8.9 , with only the patient with litigation involved scoring less than 8 .

\section{Anatomic outcome}

During final clinical follow-up (mean 21.2 months, \pm 9.7 months, range $12-36$ months), all eight patients were evaluated with postoperative MRA. No patient had a normal rotator-cuff tendon insertion. One patient demonstrated a persistent partial articular surface defect. However, the other seven patients demonstrated leakage of dye into the subacromial space, suggesting a full-thickness rotator-cuff tear (Figure 1). In all cases, the position of the needle was confirmed using fluoroscopy and a small amount of gadolinium dye. This is performed routinely by our radiologists. In no case was there inadvertent injection of the subacromial space or adjacent soft tissue (eg, subscapularis). However, in all cases with further dye injection, filling of the glenohumeral joint was followed by rapid extravasation of contrast into the subacromial space. This indicated correct positioning of the needle, but also a full-thickness rotator-cuff defect.

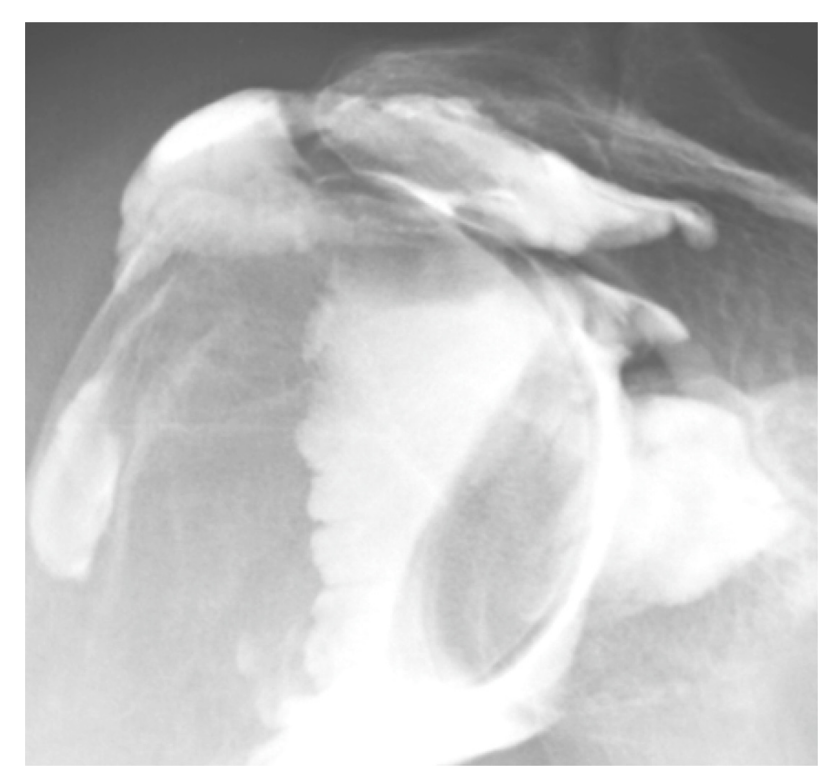

Figure I Arthrogram of a right shoulder, demonstrating dye leakage into the subacromial space indicative of a full-thickness rotator-cuff tear. 
However, in our seven patients with full-thickness rotatorcuff defects, a significant tendon discontinuity was identified in only two. In these two patients, a discontinuous tendon with intervening contrast was identified medially to the tendon insertion into the bone (Figure 2). In the other five patients, an obvious or large tendon disruption was not demonstrated on MRA. However, in all five patients, there were areas within the repair that were suspicious for a tendon defect (eg, area of thinness), which allowed dye to pass from the glenohumeral joint into the subacromial space (Figure 3). This therefore indicated a full-thickness tear. However, an actual retracted tendon defect was not apparent, due its relatively small size and imaging limitations, such as volume averaging. Due to the small number of total patients and the presence of only one patient with a completely intact rotator cuff (ie, no full-thickness defect), valid statistical correlation was not possible.

\section{Discussion}

The current retrospective study demonstrated that transtendon rotator-cuff repair of PASTA lesions can lead to excellent clinical results in this small series of patients. While the number of patients in this study is limited, there was a significant improvement in clinical outcome scores

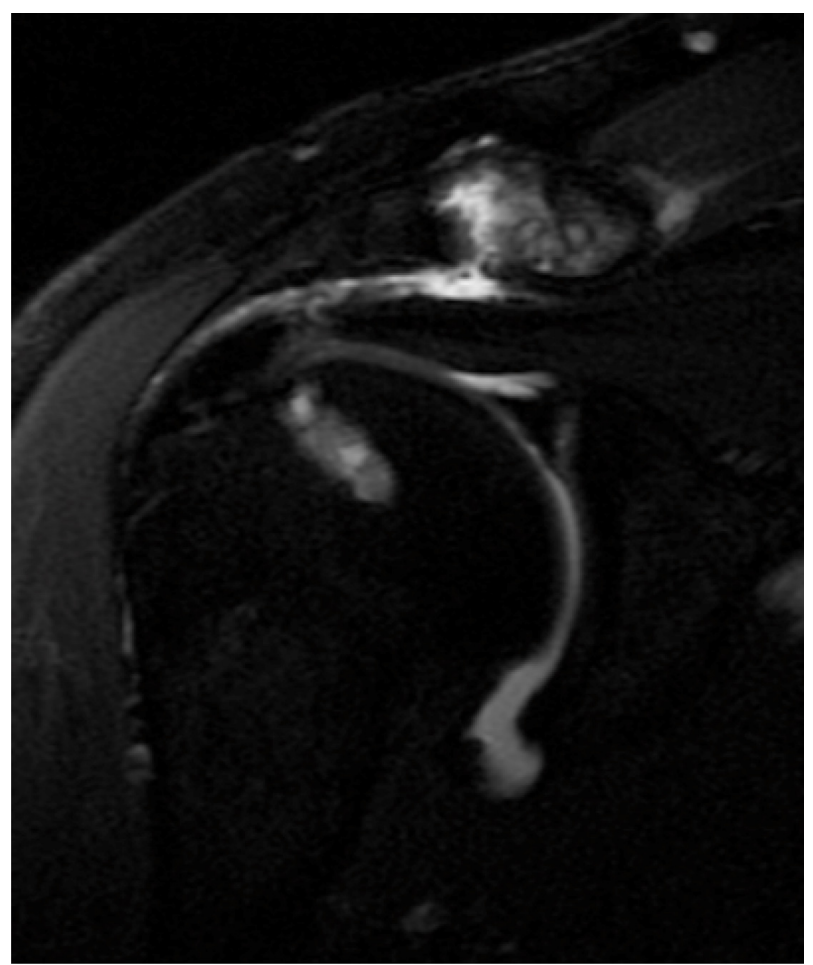

Figure 2 T2-weighted coronal magnetic resonance imaging arthrogram of a right shoulder, demonstrating a full-thickness rotator-cuff defect medial to the rotatorcuff footprint, with intervening contrast.

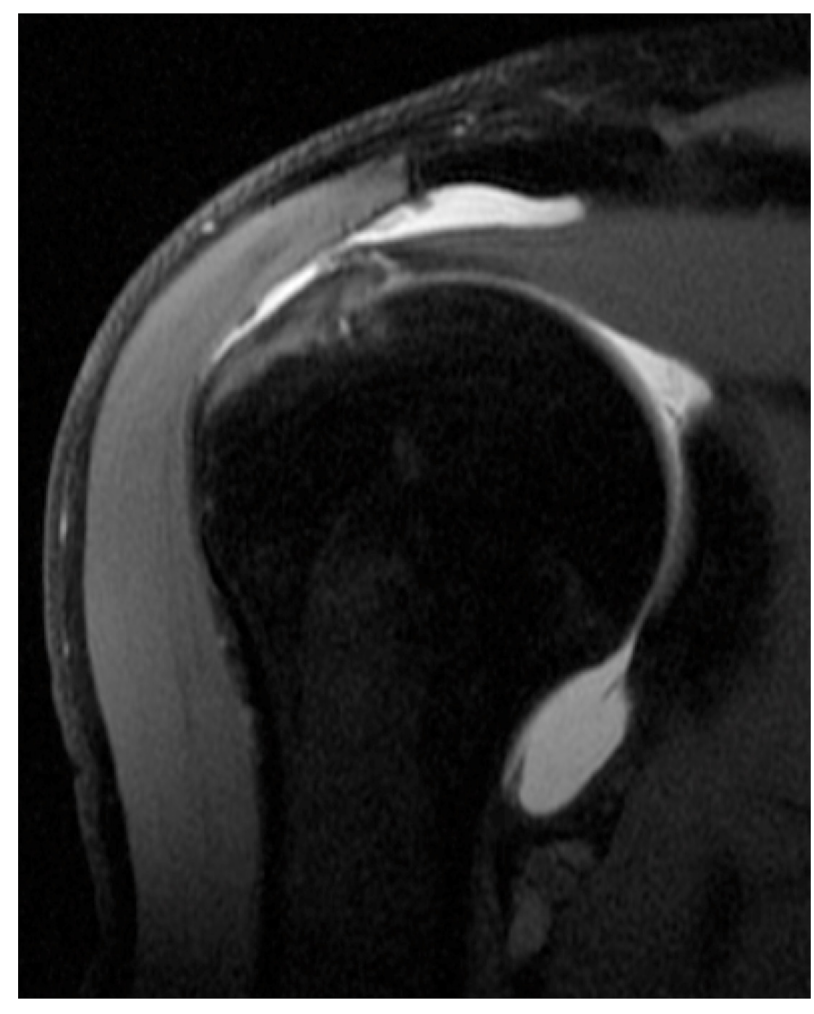

Figure 3 T2-weighted coronal magnetic resonance imaging arthrogram of a right shoulder, demonstrating a small defect suspicious for minor tendon discontinuity.

following transtendon rotator-cuff repair (only one patient demonstrated low ASES and SST scores). Other authors have found similar results when repairing articular surface lesions, whether the tear was converted to a full-thickness tear and then repaired ${ }^{10,12,13}$ or repaired using a transtendon technique..$^{11,14,15}$ To our knowledge, to date no study has compared nonoperative versus operative treatment of patients with partial-thickness rotator-cuff tears, and thus the clinical outcome of operative treatment over the natural history of partial-thickness rotator-cuff tears is unclear.

The anatomic results were extremely disappointing, with seven of eight patients having full-thickness defects through the rotator-cuff repair site based on MRA. Because of the poor anatomic results, we no longer routinely perform transtendon rotator-cuff repair in patients with high-grade $(>50 \%$ of thickness torn) PASTA lesions. Castricini et $\mathrm{al}^{9}$ reported on 33 patients following arthroscopic transtendon rotator-cuff repair. Twenty-two of their patients underwent postoperative MRI evaluation without intra-articular contrast. They had no patients with a full-thickness tear of the rotator cuff, although three patients had reduced tendon thickness.

In contrast, of the eight patients in our study who underwent postoperative MRA, seven showed dye extravasation into the subacromial space and were radiographically 
diagnosed with a full-thickness tear. We are unaware of any other study utilizing postoperative MRA to evaluate the features of transtendon repair of PASTA lesions. While the majority of patients had improved clinical outcome, the prevalence of persistent rotator-cuff defects was surprising and disappointing. The sensitivity and specificity of MRA for PASTA lesions have been reported as $84 \%$ and $96 \%$, respectively. ${ }^{15}$ Furthermore, a recent study demonstrated that MRA had a sensitivity of $96 \%$ and specificity of $99 \%$ for full-thickness rotator-cuff tears. ${ }^{22}$ Given the accuracy of MRA, these results suggest that despite repair using a transtendon technique, the majority of patients demonstrated fullthickness rotator-cuff defects despite initial partial-thickness tearing, which is likely related to the technique of repair.

Trantalis et $\mathrm{al}^{23}$ described an unusual structural mechanism of tendon failure following double-row rotator-cuff repair of full-thickness rotator-cuff tears. On follow-up MRA imaging in these patients, the footprint of the tendon appeared well fixed to the greater tuberosity, with normal thickness. However, the medial portion of the tendon was torn with full-thickness defects through the tendon medially to an intact footprint of the rotator cuff. In these patients, various full-thickness longitudinal defects were identified, medially to the tendon footprint and associated with the medial mattress sutures. These defects allowed dye to leak into the subacromial space on MRA, indicative of a full-thickness rotator-cuff tear.

We hypothesize that a similar mechanism of failure occurred in patients undergoing transtendon repair of partial-thickness tears. In these patients, similarly to patients undergoing double-row rotator-cuff repair, the medial mattress sutures absorb the majority of the load during early rehabilitation. During cyclic loading, failure of the tendon repair occurs initially at the medial mattress sutures, with the mattress sutures pulling through the tendon medially to the repair site. ${ }^{24}$ This can eventually lead to a full-thickness defect through the rotator cuff.

However, of the seven patients with reported fullthickness rotator-cuff defects, only one patient was dissatisfied with the procedure. This one patient, who stated they would still have surgery again, was involved in an ongoing litigation claim. The other six patients reported improvements in subjective and objective outcome measures. Therefore, the short-term clinical relevance of a small full-thickness defect through the medial mattress sutures is unclear, as is the natural history of these longitudinal defects. In this young patient population, any full-thickness defect, even those that are relatively small, should raise concerns, due to the potential of tear progression, both symptomatically and silently.
When surgeons are faced with a PASTA lesion, the optimal surgical treatment remains controversial. Transtendon rotator-cuff repair has the theoretical advantages of maintaining a viable and robust lateral tendon, potentially leading to a more anatomic reconstruction of the rotator-cuff footprint. Furthermore, recent biomechanical studies have demonstrated the superior mechanical properties of a transtendon rotator-cuff repair when compared to a double-row rotatorcuff repair. ${ }^{25}$

Due to the poor anatomic outcomes, the senior author has now altered his indications for a transtendon repair. In this small series, all patients had $>50 \%$ of the thickness torn (mean 62.1\%), which may have contributed to the disappointing anatomic results. During surgery, we believe it is important to assess the integrity of the remaining intact tendon when considering a transtendon repair. While this can be difficult to determine, clinically careful evaluation of the quality of the remaining tissue, as well as an estimation of the amount of tendon thickness torn, is paramount. Where over $50 \%$ of the tendon is torn or the quality of the remaining tendon is poor, we do not recommend routinely performing a transtendon rotator-cuff repair. In contrast, completion of the tear with double-row rotator-cuff repair is our technique of choice. This technique reconstructs the footprint of the rotator cuff, and may minimize medial defects. A double-row footprint reconstruction technique is now performed in the majority of partial-thickness tears requiring repair, and may improve the prevalence of intact rotator-cuff repairs. ${ }^{26}$

We now reserve transtendon repairs to rare cases of younger patients where $33 \%-50 \%$ of the tendon is torn, there are significant symptoms related to loss of strength, and a repair (as opposed to a debridement) is desired. In this situation, there remains a large amount of robust lateral tendon that can be preserved, which may potentially minimize medial defects.

When a transtendon repair is being considered, a number of technical factors may also be borne in mind. These include minimizing trauma to the remaining rotator-cuff tissue (eg, small implants, small suture-passing instruments, avoiding oblique suture passage), avoiding overtensioning of the repair (eg, avoiding excessive medial suture passage), and creating wide mattress stitches. Furthermore, when using two anchors, tying sutures between anchors in a doublepulley fashion ${ }^{14}$ creates the largest mattress suture possible, and may minimize suture pull-through. Finally, creating a suture-bridge construct utilizing the residual suture limbs of the medial mattress sutures may be considered. In larger tears, this may provide further compression of the tendon 
against the footprint, and may more evenly distribute load across multiple anchors, potentially minimizing suture pullthrough.

This study was a retrospective case series and has a number of inherent limitations. It had a relatively small sample size, which may have introduced bias into the study, limiting its statistical strength. The follow-up period was relatively short, and we did not have a control group to make comparative inferences. Furthermore, six patients were lost to follow-up and were not included in the study. Four patients had incomplete clinical follow-up, and two patients refused postoperative imaging using MRA. The impact of these patients lost to follow-up should be strongly considered. However, within the purpose and scope of the study, we still believe that the results are clinically meaningful. We believe our results are significant, should be taken into consideration when treating patients with PASTA lesions, and provide direction for further research.

\section{Conclusion}

Transtendon repair of PASTA lesions may lead to improvements in clinical outcome. However, there was a high incidence of full-thickness rotator-cuff defects following repair based on MRA follow-up.

\section{Acknowledgment}

Financial support for this study was received from the Canadian Orthopaedic Foundation (Hip Hip Hooray! program).

\section{Disclosure}

The authors report no conflicts of interest in this work.

\section{References}

1. Malabar F, Krieger RE. Rupture of the supraspinatus tendon and other lesions in and about the subacromial bursa. In: Codman EA, editor. The Shoulder. Boston: Thomas Todd; 1934.

2. Fukuda H, Mikasa M, Yamanaka K. Incomplete thickness rotator cuff tears diagnosed by subacromial bursography. Clin Orthop Relat Res. 1987;(223):51-58.

3. Snyder SJ. Arthroscopic classification of rotator cuff lesions and surgical decision making. In: Shoulder Arthroscopy. 2nd ed. Philadelphia: Lippincott Williams \& Wilkins; 2003:201-207.

4. Spencer Jr. EE. Partial-thickness articular surface rotator cuff tears: An all-inside repair technique. Clin Orthop Relat Res. 2010;468(6): $1514-1520$.

5. Wolff AB, Sethi P, Sutton KM, Covey AS, Magit DP, Medvecky M. Partial-thickness rotator cuff tears. J Am Acad Orthop Surg. 2006;14(13):715-725.

6. Cordasco FA, Backer M, Craig EV, Klein D, Warren RF. The partialthickness rotator cuff tear: is acromioplasty without repair sufficient? Am J Sports Med. 2002;30(2):257-260.

7. Matava MJ, Purcell DB, Rudzki JR. Partial-thickness rotator cuff tears. Am J Sports Med. 2005;33(9):1405-1417.
8. Weber SC. Arthroscopic debridement and acromioplasty versus mini-open repair in the treatment of significant partial-thickness rotator cuff tears. Arthroscopy. 1999;15(2):126-131.

9. Castricini R, Panfoli N, Nittoli R, Spurio S, Pirani O. Transtendon arthroscopic repair of partial-thickness, articular surface tears of the supraspinatus: results at 2 years. Chir Organi Mov. 2009;93 Suppl 1: S49-S54.

10. Deutsch A. Arthroscopic repair of partial-thickness tears of the rotator cuff. J Shoulder Elbow Surg. 2007;16(2):193-201.

11. Ide J, Maeda S, Takagi K. Arthroscopic transtendon repair of partialthickness articular-side tears of the rotator cuff: anatomical and clinical study. Am J Sports Med. 2005;33(11):1672-1679.

12. Iyengar JJ, Porat S, Burnett KR, Marrero-Perez L, Hernandez VH, Nottage WM. Magnetic resonance imaging tendon integrity assessment after arthroscopic partial-thickness rotator cuff repair. Arthroscopy. 2011;27(3):306-313.

13. Kamath G, Galatz LM, Keener JD, Teefey S, Middleton W, Yamaguchi K. Tendon integrity and functional outcome after arthroscopic repair of high-grade partial-thickness supraspinatus tears. J Bone Joint Surg Am. 2009;91(5):1055-1062.

14. Lo IK, Burkhart SS. Transtendon arthroscopic repair of partialthickness, articular surface tears of the rotator cuff. Arthroscopy. 2004;20(2):214-220.

15. Meister K, Thesing J, Montgomery WJ, Indelicato PA, Walczak S, Fontenot W. MR arthrography of partial thickness tears of the undersurface of the rotator cuff: an arthroscopic correlation. Skeletal Radiol. 2004;33(3):136-141.

16. Koh KH, Shon MS, Lim TK, Yoo JC. Clinical and magnetic resonance imaging results of arthroscopic full-layer repair of bursal-side partial-thickness rotator cuff tears. Am J Sports Med. 2011;39(8): 1660-1667.

17. Seo YJ, Yoo YS, Kim DY, Noh KC, Shetty NS, Lee JH. Trans-tendon arthroscopic repair for partial-thickness articular side tears of the rotator cuff. Knee Surg Sports Traumatol Arthrosc. 2011;19(10):1755-1759.

18. Stuart KD, Karzel RP, Ganjianpour M, Snyder SJ. Long-term outcome for arthroscopic repair of partial articular-sided supraspinatus tendon avulsion. Arthroscopy. 2013;29(5):818-823.

19. Kim KC, Shin HD, Cha SM, Park JY. Clinical outcomes after arthroscopic trans-tendon suture-bridge technique in partial-thickness articular-side rotator cuff tear. Knee Surg Sports Traumatol Arthrosc. 2013;21(5):1183-1188.

20. Richards RR, An KN, Bigliani LU, et al. A standardized method for the assessment of shoulder function. J Shoulder Elbow Surg. 1994;3(6): 347-352.

21. L'Insalata JC, Warren RF, Cohen SB, Altchek DW, Peterson MG. A self-administered questionnaire for assessment of symptoms and function of the shoulder. J Bone Joint Surg Am. 1997;79(5):738-748.

22. Waldt S, Bruegel M, Mueller D, et al. Rotator cuff tears: assessment with MR arthrography in 275 patients with arthroscopic correlation. Eur Radiol. 2007;17(2):491-498.

23. Trantalis JN, Boorman RS, Pletsch K, Lo IK. Medial rotator cuff failure after arthroscopic double-row rotator cuff repair. Arthroscopy. 2008;24(6):727-731.

24. Mazzocca AD, Millett PJ, Guanche CA, Santangelo SA, Arciero RA. Arthroscopic single-row versus double-row suture anchor rotator cuff repair. Am J Sports Med. 2005;33(12):1861-1868.

25. Gonzalez-Lomas G, Kippe MA, Brown GD, et al. In situ transtendon repair outperforms tear completion and repair for partial articularsided supraspinatus tendon tears. J Shoulder Elbow Surg. 2008;17(5): 722-728.

26. Millett PJ, Warth RJ, Dornan GJ, Lee JT, Spiegl UJ. Clinical and structural outcomes after arthroscopic single-row versus double-row rotator cuff repair: a systematic review and meta-analysis of level I randomized clinical trials. J Shoulder Elbow Surg. 2014;23(4):586-597. 
Open Access Journal of Sports Medicine

Dovepress

\section{Publish your work in this journal}

Open Access Journal of Sports Medicine is an international, Visit http://www.dovepress.com/testimonials.php to read real quotes peer-reviewed, open access journal publishing original research, from published authors.

reports, reviews and commentaries on all areas of sports

medicine. The manuscript management system is completely

online and includes a very quick and fair peer-review system.

Submit your manuscript here: http://www.dovepress.com/open-access-journal-of-sports-medicine-journal 